\title{
Über die Wirkung des Strychnins auf die absolute Kraft des Froschherzens.
}

\author{
Von \\ Itaro Kikuchi. \\ (㐘地伊太郎) \\ (Aus dem pharmakologischen Institut von Prof. S. Yagi, \\ Tohoku Universität $\approx u$ Sendai.)
}

Die therapeutischen Erfolge des Strychnins bei Zirkulationsstörungen sind von den meisten als auf seiner Wirkung auf die vasomotorischen Zentren beruhend angenommen worden, da alle diesbezüglichen experimentellen Untersuchungen nur seine anregende Wirkung auf diese Zentren bewiesen haben, ohne dass man eine günstige Wirkung auf das Herz selbst hätte nachweisen kömnen. Denken wir aber daran; dass Strychnin nicht nur auf das Zentralnervensystem, sondern auch auf die peripheren Organe, wie den Magen und Darm, anregend wirkt, so scheint es voreilig zu sein, seine günstige Herzwirkung zu verneinen, ehe diese nicht von verschiedenen Richtungen her ausgeschlossen wird. Diebisherigen Untersuchungen haben sich aber fast nur mit dem Rhythmus und der Amplitüde des Herzens beschäftigt, ohne die absolute Kraft des Herzens zu berücksichtigen. Nenerdings hat Junkmann ${ }^{12}$ die Wirkung des Strychnins auf die Froschherzdynamik untersucht und daraus geschlossen, dass es Abnahme der isometrischen Spannungsmaxima herbeiführe, ohne dabei die isometorischen Kontraktionen zu beeinflussen. Doch scheint sein Experiment für solche Schlussfolgerung nicht zu genügen, da die Abnahme der isometrischen Spannungsmaxima keineswegs die konstante Folge ist und selbst bei positiven Fällen die Versuche im Anschluss an andere ausgeführt worden sind, so dass es schwierig zu unterscheiden ist, ob die Abnahme als echte Folge der Strychninwirkung anzusehen ist oder nicht. Das hat mich veranlasst, die Wirkung des Strychnins auf die absolute Kraft des Herzens zu untersuchen.

Meine Untersuchungen wurden ausschliesslich am Herzen der Rana japonica in situ, und zwar nach der von mir früher beschriebenen Me-

1) Junkmann, Arch. f. exp. Path. u. Pharm., 1925, 105, 169. 
thode ${ }^{1}$ ausgeführt. Strychnin wurde als Nitrat oder Sulfat gebraucht, ohne dass aber irgend ein Unterschied in den Versuchsresultaten zwischen den beiden auftrat. Die Versuchsergebnisse sind folgende :

Bei der Durchströmung des Herzens mit Strychninlösung führten Konzentrationen unter 1:500,000 keine bemerkbaren Veränderungen der absoluten Kraft und des Minutenvolums herbei. Konzentrationen von

\begin{tabular}{|c|c|c|c|c|c|c|c|c|c|c|c|c|c|c|c|c|}
\hline \multirow{3}{*}{ 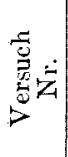 } & \multirow{3}{*}{ 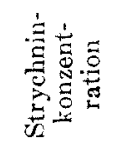 } & \multicolumn{15}{|c|}{ Absolute $\mathrm{Kraft}$ in $\mathrm{cm} \mathrm{H}_{2} \mathrm{O}$} \\
\hline & & \multirow{2}{*}{\multicolumn{3}{|c|}{$\begin{array}{l}\text { Ringer- } \\
\text { perioden }\end{array}$}} & \multicolumn{12}{|c|}{ Strychninperioden } \\
\hline & & & & & $5^{\prime}$ & $10^{\prime}$ & $15^{\prime}$ & $20^{\prime}$ & $25^{\prime}$ & $30^{\prime}$ & $35^{\prime}$ & $40^{\prime}$ & $45^{\prime}$ & $50^{\prime}$ & $55^{\prime}$ & $60^{\prime}$ \\
\hline 10 & $1: 500,000$ & & 57 & 57 & 57 & 57 & 57 & 56 & 56 & 55 & 55 & 55 & 55 & 54 & 54 & 54 \\
\hline 15 & $1: 250,000$ & 56 & 56 & 55 & 60 & 60 & 62 & 62 & 60 & 58 & 55 & 54 & 54 & 53 & 58 & 52 \\
\hline 20 & $1: 100,000$ & 56 & 56 & 56 & 65 & 65 & 64 & 64 & 63 & 63 & 62 & 61 & 61 & 60 & 60 & \\
\hline 22 & $1: 100,000$ & 54 & 53 & 53 & 55 & 58 & 59 & 58 & 58 & 56 & 56 & 55 & 55 & & & \\
\hline 19 & $1: 50,000$ & & 53 & 53 & 63 & 64 & 64 & 62 & 61 & 62 & 61 & 60 & 59 & 58 & 58 & \\
\hline 24 & $1: 50,000$ & & 63 & 63 & 69 & 69 & 70 & 69 & 68 & 68 & 68 & 67 & 67 & 67 & 66 & \\
\hline 21 & $1: 50,000$ & 50 & 50 & 50 & 55 & 56 & 54 & 53 & 52 & 52 & 52 & 52 & 51 & 51 & 50 & \\
\hline 25 & $1: 25,000$ & & 63 & 63 & 68 & $6 \mathrm{~s}$ & 65 & 64 & 60 & 56 & 56 & 53 & 49 & & & \\
\hline 28 & $1: 25,000$ & 67 & 67 & 66 & 70 & 71 & 69 & 68 & 66 & 63 & 61 & 60 & 59 & 59 & & \\
\hline 18 & $1: 10,000$ & 67 & 66 & 66 & 64 & 65 & 58 & 57 & 48 & 48 & $4 \overline{7}$ & 45 & 43 & & & \\
\hline
\end{tabular}

$1: 250,000-1: 50,000$, welche beim Straubschen Versuche auf die Amplitüde und Pulsfrequeuz des Herzens keine irgendwie nachweisbare Wirkung entfalteten, übten deutliche Zunahme der absoluten Kraft aus, wobei das Minutenvolum nur selten leichte Zunahme zeigte, in den meisten Fällen aber unverändert blieb. Bei einer noch gesteigerten Konzentration, wie $\mathbf{1}: \mathbf{2 5}$, 000 , welche keine nach weisbaren Veränderungen der Amplitüde und der Pulsfrequenz bewirkte, wenn sie auch leichte Zunahme der ersteren und geringfügige Verminderung der letzteren gelegentlich hervorriefen, sank die

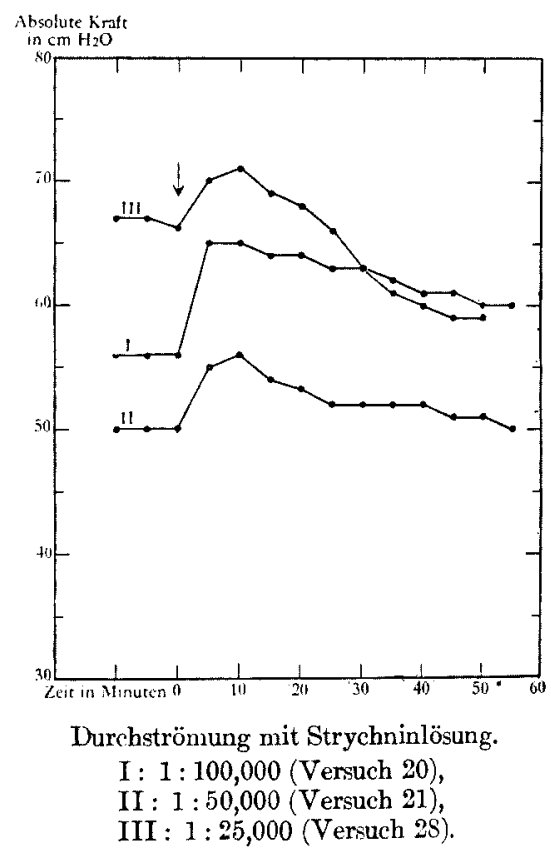

1) Kikuchi, Tohoku J. Exp. Med., 1928, 11, 116. 
absolute Kraft nach vorïbergehender dentlicher Steigerung beständig. Bei stark konzentrierter Lösung, d. h. über 1 : 10,000, kamen deutliche Abnahme der absoluten Kraft, der Amplitüde und der Pulsfrequenz von Anfang an zum Vorschein (Tabelle). Zur besseren Übersicht lasse jch eine graphische Darstellung einiger Versuche mit verschiedenem Verlauf folgen.

Daraus ersehen wir, dass Strychnin in geeigneten Konzentrationen erhebliche Steigerung der absoluten Kraft des Froschherzens hervorruft. Diese Wirkung mag auch bei den therapeutischen Erfolgen des Strychnins eine Rolle spielen. 